IdeAs

Idées d'Amériques

9 | 2017

Poètes et éditeurs : diffuser la poésie d'avant-garde américaine (depuis 1945)

\title{
Claude Chastagner, Patti Smith : Outside
}

Montpellier, Presses universitaires de la Méditerranée, 2015, 169p.

Johanna Nepote-Cit

\section{(c) OpenEdition}

\section{Journals}

Édition électronique

URL : https://journals.openedition.org/ideas/1919

DOI : 10.4000/ideas.1919

ISSN : 1950-5701

Éditeur

Institut des Amériques

Référence électronique

Johanna Nepote-Cit, «Claude Chastagner, Patti Smith : Outside », IdeAs [En ligne], 9 | 2017, mis en ligne le 12 juillet 2017, consulté le 19 octobre 2022. URL : http://journals.openedition.org/ideas/1919 ; DOI : https://doi.org/10.4000/ideas.1919

Ce document a été généré automatiquement le 19 octobre 2022.

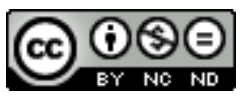

Creative Commons - Attribution - Pas d'Utilisation Commerciale - Pas de Modification 4.0 International - CC BY-NC-ND 4.0

https://creativecommons.org/licenses/by-nc-nd/4.0/ 


\section{Claude Chastagner, Patti Smith : Outside}

Montpellier, Presses universitaires de la Méditerranée, 2015, 169p.

Johanna Nepote-Cit

\section{RÉFÉRENCE}

Claude Chastagner (dir.), Patti Smith: Outside, Montpellier, Presses universitaires de la Méditerranée, 2015, 169p.

1 Comment aborder le personnage de Patti Smith, musicienne, poète, photographe, activiste, et grande prêtresse de la scène punk américaine? Outside rassemble huit articles et une bibliographie sélective dont l'ambition est précisément de présenter au lecteur quelques-unes des facettes de la punk poet laureate.

2 Dans son introduction, Claude Chastagner évoque le paradoxe Patti Smith, paradoxe nourri par le temps et le succès : la jeune punk qui se disait «en dehors de la société » dans Rock'n'Roll Nigger peut-elle prétendre aujourd'hui au même statut qu'à ses débuts ? Quelque quarante ans plus tard, après avoir reçu nombre de prix et de distinctions et avoir vendu beaucoup d'albums, ne devrait-on pas la considérer comme un pilier de l'establishment?

3 Carrie Noland étudie avec minutie le lien entre Patti Smith et Arthur Rimbaud. Si elle est loin d'être la seule, dans les années 70, à tenter de vivre le « Mythe de Rimbaud", Patti Smith en fait une affaire très personnelle, puisqu'elle va, en 2016, jusqu'à acheter une maison ayant appartenu à la famille Rimbaud. Noland s'attache à décortiquer les textes, afin de montrer la présence de Rimbaud dans les choix stylistiques de Smith.

4 Amélie Ducroux s'interroge quant à elle sur ce qui a pu pousser la poète Smith vers le rock, par le biais d'une étude systématique des textes de l'artiste et d'une réflexion sur la voix (trouver sa voix), et en interrogeant Just Kids, les mémoires de Smith qui 
retracent notamment sa relation avec Robert Mapplethorpe et ses premières années à New York, lorsque s'opère le basculement vers le rock.

Ulara Toma s'attache au(x) genre(s) de Patti Smith. Les photographies de Smith par Mapplethorpe ont fait connaitre son style androgyne. Toma part de ce premier constat qu'est le goût de Smith pour le travestissement pour montrer qu'aux yeux de l'artiste, le genre peut se porter ou se quitter comme on le ferait d'un vêtement. Elle évoque ensuite des témoignages de Smith, parlant de la manière dont elle s'est efforcée, lorsqu'elle l'a souhaité, d'apprendre la féminité, et la façon dont elle s'y est prise, pour élargir sa réflexion à Patti Smith comme personnage théâtral, tant sur scène que dans ses textes.

Simon Warner s'intéresse au lien qui unit Patti Smith à la Beat Generation, lien de filiation artistique mais aussi d'amitié, en particulier avec Allen Ginsberg, lien souvent évoqué, certes, mais aussi souvent grossièrement résumé aux seuls choix vestimentaires de Smith, à la facilité avec laquelle elle se donne des héros, et sa volonté de s'inscrire dans ce mouvement qu'elle admire. Au point que sa participation au documentaire One Fast Move or I'm Gone, Kerouac's Big Sur fut très critiquée par trois chroniqueurs. Pour les biographes cités par Warner, le lien est plus profond et se décèle par exemple dans la vision qu'a Patti Smith du poète comme performer. Warner donne dans une première partie de son article ce tour d'horizon, puis présente les réponses à un questionnaire qu'il a proposé à une vingtaine de spécialistes de la musique rock, de Patti Smith, ou encore de la Beat Generation, afin de réactualiser la question et de recueillir les avis les plus récents.

7 Richard Elliott choisit, pour sa part, d'explorer les thèmes de l'aventure, de la mémoire, et du retour dans Banga, un album récent de Patti Smith. A l'aventure narrative, présente dès le premier titre, "Amerigo ", se joint l'aventure de l'expérimentation musicale. Les thèmes de mémoire et de retour sont abordés ensuite. La réflexion est d'abord contextualisée : il note la sortie de plusieurs biographies de Patti Smith qui la consacrent au début des années 2000, lesquelles érigent son premier album Horses au rang d'œuvre suprême et inégalable. Elliott analyse d'abord Horses, brièvement, afin de montrer les fils qui s'en dégagent et que Patti Smith continue, selon lui, de tisser.

Rachel Kessler se concentre sur l'art plastique de Patti Smith, en particulier sur ses aspects corporels et cinétiques. Elle évoque William Blake et Antonin Artaud comme principales influences. La création artistique, même visuelle, est inséparable du verbe, quand l'écriture est catalyseur de l'invention. A telle enseigne que Patti Smith, selon Kessler, s'entraînera à imiter l'écriture de Blake, comme si le mouvement accompli en laissant sa main retracer cette trajectoire vieille de quelque deux cents ans était un préambule nécessaire, selon la critique.

9 Le dernier article, qui précède la bibliographie sélective très à jour, est en réalité une partie d'un entretien donné par Lenny Kaye, le guitariste de Patti Smith, réalisé par Stan Cuesta. Il y parle de son propre rapport à la musique, et de Patti Smith elle-même, de leurs débuts, lorsque Patti Smith écrivait des textes qu'elle déclamait, et qu'il l'accompagnait à la guitare, de la création du groupe, mais aussi du processus de composition et d'écriture. Les souvenirs et observations du musicien fournissent un angle de perception intéressant sur l'œuvre et sur la pratique musicale et créatrice de Patti Smith, et le lecteur s'amusera peut-être d'y retrouver des éléments mis en évidence, dans une perspective plus théorique, dans les autres articles de l'ouvrage. Ce lien entre éléments concrets, parfois même biographiques, et une vision plus abstraite 
et conceptuelle est l'un des points forts de cet ouvrage qui élargit les perspectives interprétatives sur l'œuvre, le personnage et peut-être la personne qu'est Patti Smith.

\section{AUTEURS}

\section{JOHANNA NEPOTE-CIT}

Agrégée d'anglais, doctorante Université Paris-Sorbonne 